\title{
Pigmentary Glaucoma refractory to medical therapy (Clinical Image)
}

\author{
Kirti Singh, Mainak Bhattacharyya*, Annu Joon, Sumit Kumar and Nikhil Gotmare \\ Department of Opthamology, Guru Nanak Eye Centre, India \\ *Corresponding author:Mainak Bhattacharyya, Guru Nanak Eye Centre, Maharaja Ranjit Singh Marg, Delhi, India
}

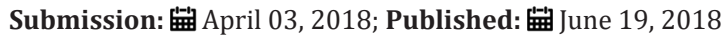

\begin{abstract}
Pigmentary glaucoma (PG) a type of secondary open angle glaucoma is characterized by classic diagnostic triad of Krukenberg's spindle; mid peripheral iris transillumination defects and dense Trabecular pigmentation. Treatment consists of medical management followed by laser iridotomy/ laser trabeculoplasty and trabeculectomy in recalcitrantcases; trabeculectomy is challenging with a higher risk of bleb failure and Hypotony Maculopathy.

We report the case of a 43-year-old one-eyed male with BCVA 6/9 OS (log MAR 0.2); intraocular pressure (IOP) 34mmHg, oedematous cornea, prominent Krukenberg spindle and a very prominent Eggers line. He had advanced glaucomatous cupping (CDR 0.7:1); gonioscopy showed widely open angles, with "queer" iris configuration, and heavily pigmented PTM (Gd IV) with advanced damage on perimetry. As patient couldn't be weaned off systemic anti-glaucoma medications, trabeculectomy with antifibrotic augmentation was done. The surgery was uneventful and resulted in stabilizing IOP at 12-14 mmHg over a 6 month follow up till date.
\end{abstract}

Keywords: Pigmentary Glaucoma; Pigment Epithelium; Krukenberg Spindle; Egger's Line; Trabeculectomy; Transillumination; Iridotomy; Trabeculoplasty; Maculopathy; Oedematous; Gonioscopy; Lens Zonules; Biomicroscopy; Hypotony; Penetrance; Autosomal

Abbreviations: PG: Pigmentary Glaucoma; IOP: Intraocular Pressure; IPE: Iris Pigment Epithelium

\section{Background}

Pigmentary glaucoma (PG) typically seen in a young myopic male, is a type of secondary open angle glaucoma characterized by disruption of the iris pigment epithelium (IPE) and deposition of dispersed pigment granules throughout the anterior segment. The classic diagnostic triad consists of Krukenberg spindle; midperipheral iris transillumination defects and dense trabecular pigmentation. The iris insertion is typically posterior; peripheral

\section{Presentation}
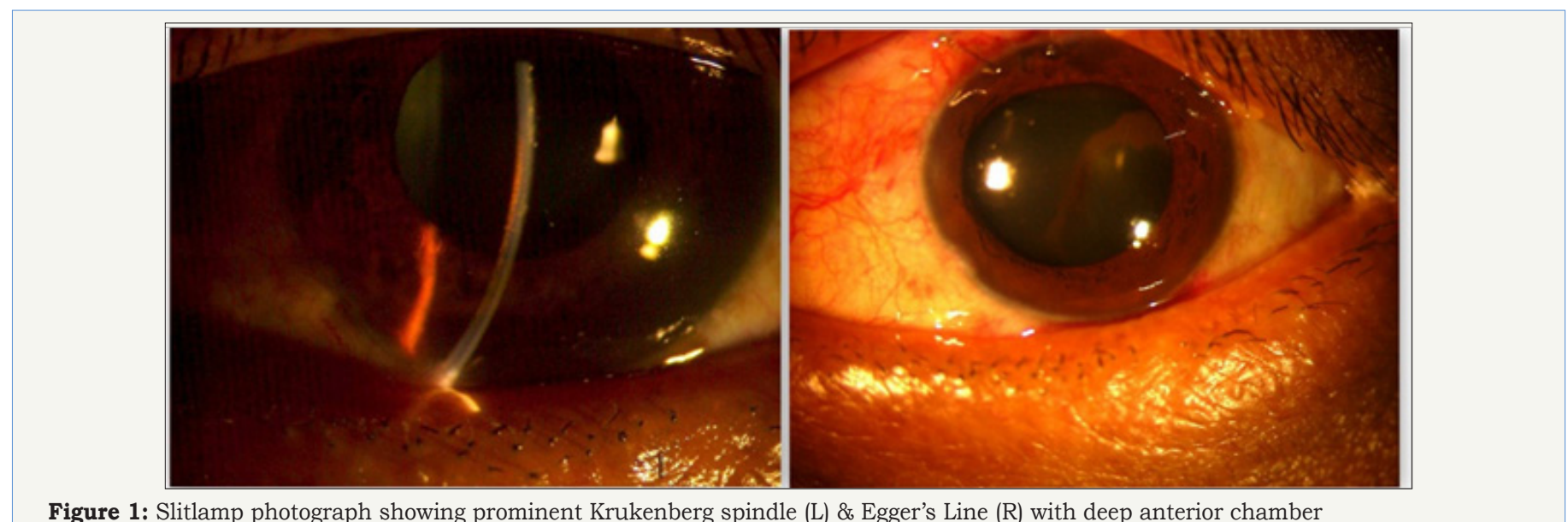

Figure 1: Slitlamp photograph showing prominent Krukenberg spindle (L) \& Egger's Line (R) with deep anterior chamber

iris tends to have a concave configuration earlier known as queer configuration [1]. Postulated mechanism of pigment dispersion is rubbing off iris pigment epithelium due to constant contact with lens zonules, possibly associated with an inherent fragility of the pigment epithelium. Aqueous outflow obstruction results due to accumulation of pigment granules in trabecular meshwork, followed by denudation, collapse, and sclerosis of trabecular beams [2]. 
A one eyed 43-year-old male presented with complaint of narrowing of peripheral fields noted for last 1 month. On presentation, his visual acuity was 6/9 OS (logMAR 0.2); intraocular pressure (IOP) was $34 \mathrm{mmHg}$ (GAT) (CCT $515 \mu \mathrm{m}$ ) and PL negative OD. Right eye was atrophic, subsequent to a childhood ocular trauma. Slit lamp biomicroscopy revealed oedematous cornea, prominent Krukenberg spindle on corneal endothelium, deep anterior chamber (ACD 3.5mm), mid dilated pupil, and a very prominent Eggers line (pigment granule deposits on hyaloidcapsular ligament) (Figure 1). Fundus evaluation revealed myopic disc with advanced glaucomatous cupping (CDR 0.7:1) with superior notching \& inferior NRR thinning (DDLS 4) (Figure 2). Gonioscopy showed widely open angles, with CBB visible 360 degrees; "queer" iris configuration, Sampaolesi's line along with heavily pigmented PTM (Gd IV) in all quadrants. (Figure 3).

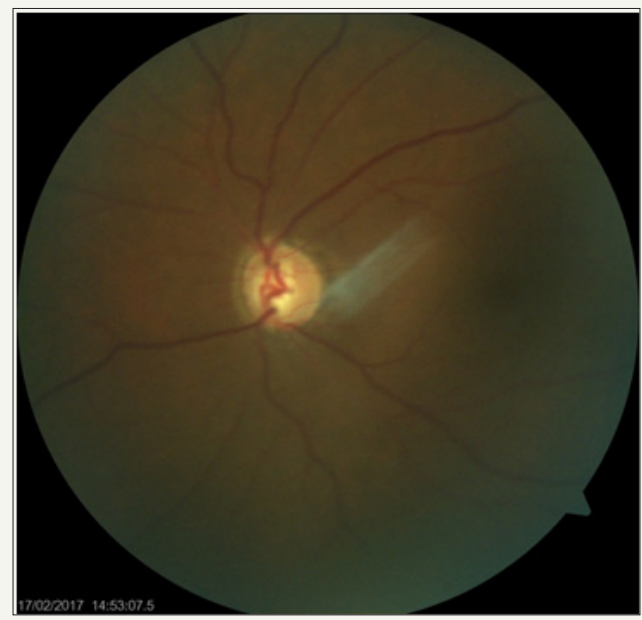

Figure 2: Fundus photograph OS showing glaucomatous optic atrophy with superior notching and inferior NRR thinning

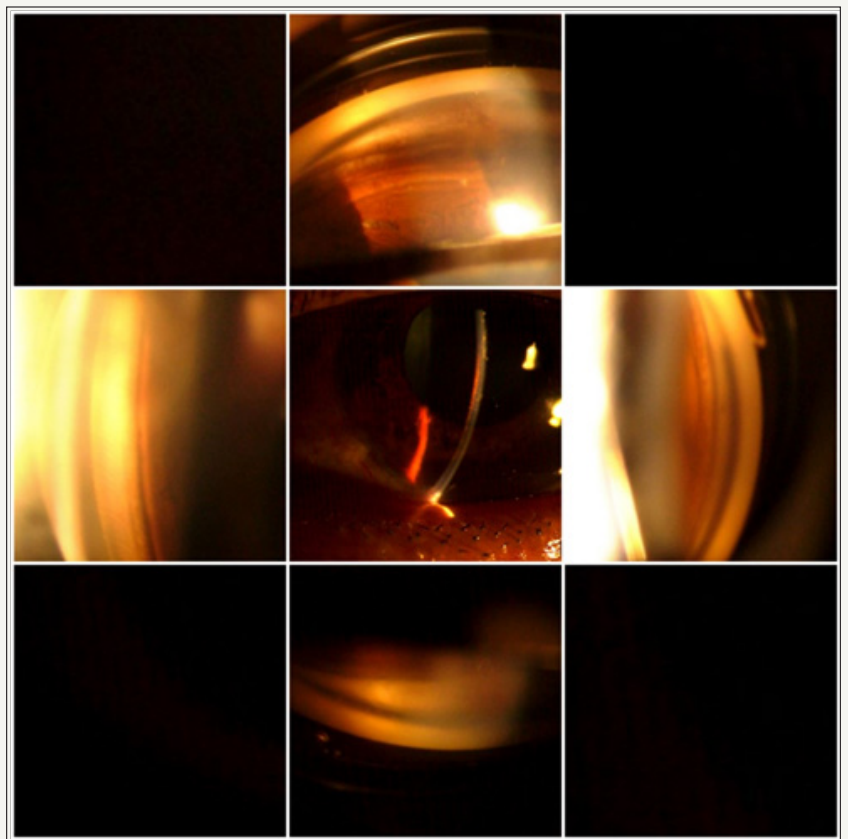

Figure 3: Gonio photographs showing widely open angles, with concave iris, Sampaolesi line and heavily pigmented TM

Visual field examination (24-2, Humphrey visual analyzer) revealed advanced field loss Bjerrum's scotoma encroaching on macula (MD: -31.03 dB, VFI 14\%); subsequently macular split was confirmed on 10-2 strategy which showed only temporal island of field left intact (Figure 4) After medical control of IOP using hyperosmotic agents and topical medications (including pilocarpine $2 \%$ ), the patient was subjected to laser iridotomy. The IOP was controlled to mid 20's, but patient could not be weaned off systemic medications (Acetazolamide). Trabeculectomy with antifibrotic augmentation $(0.1 \%$ Mitomycin $\mathrm{C}$ and amniotic membrane) was performed under all precautions, as per protocol for advanced glaucoma cases (Figure 5.1). The surgery was uneventful and resulted in stabilizing IOP at 12-14mmHg over a 6 month follow up till date. The releasable sutures were removed in graded fashion and final bleb morphology after conjunctival suture removal was E4V2H2 (IBAGS classification) (Figure 5.2). Pre-op visual acuity was regained at 4 weeks and maintained till last follow up. 


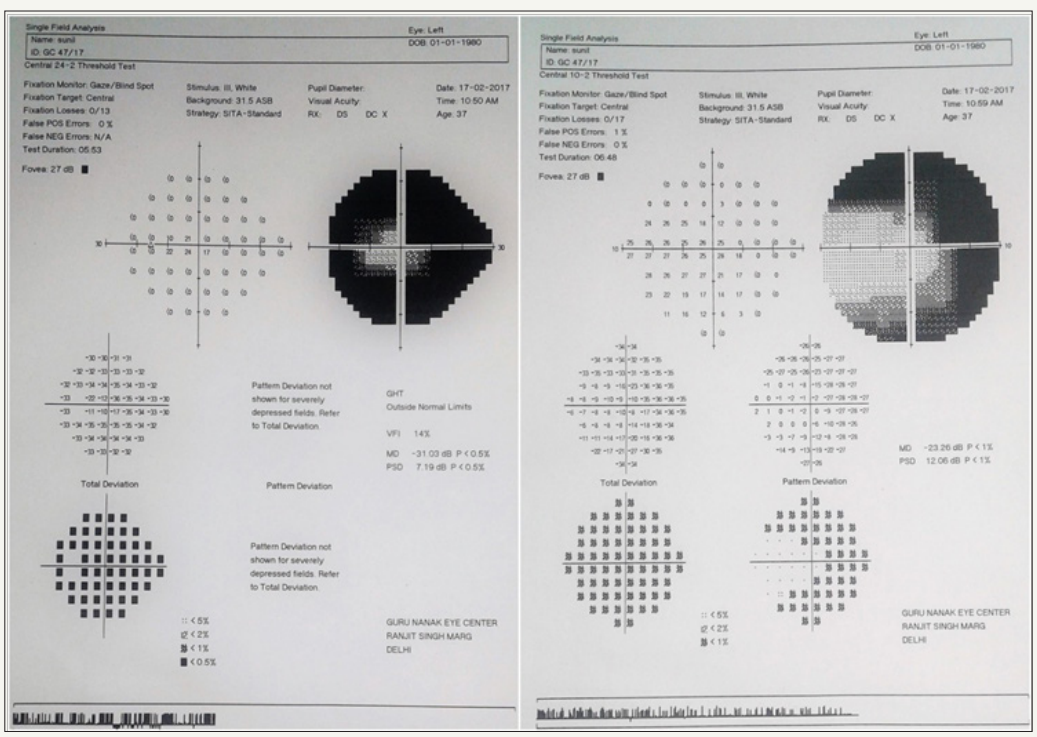

Figure 4: VF (24-2) showing advanced field loss and (10-2) depicting macular split with only temporal island spared

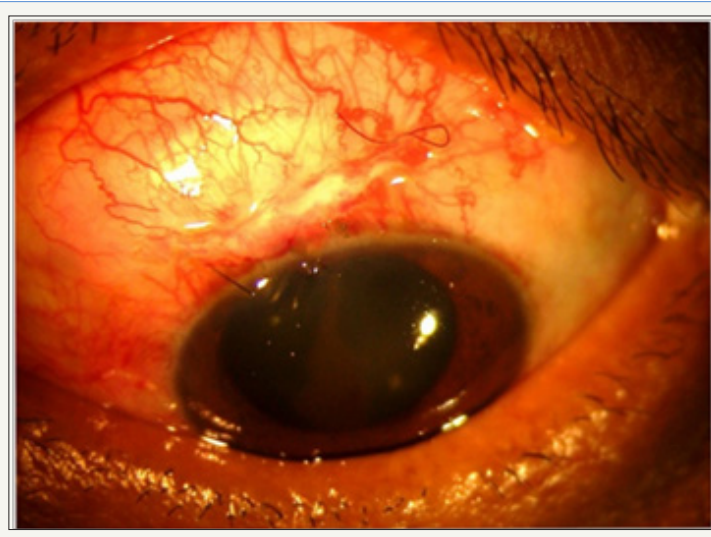

Figure 5.1:

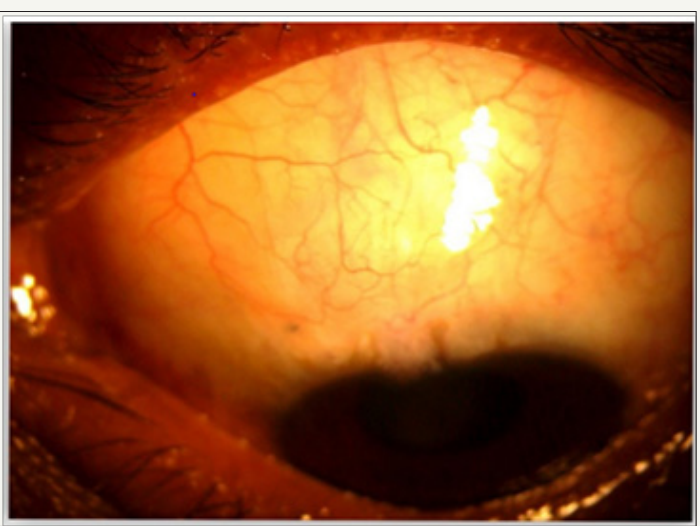

Figure 5.2: Healthy functional Bleb (E2V2H2) in the early post-operative period (L) and at 6 months follow up (R)

\section{Discussion}

Pigmentary glaucoma (PDG) is a bilateral, autosomal dominant disorder with incomplete penetrance, gene locus on chromosome

7q35-36.3 [3]. Topical medications followed by peripheral iridotomy is recommended treatment with advice to avoid excessive exercise which causes IOP spikes due to sympathetic drive induced pupil mydriasis generating increased pigment dispersal. Trabeculectomy is challenging with a higher risk of bleb failure and hypotony maculopathy. Reported age of patients with PDG requiring surgery is nearly 15 years younger than patients of OAG [4].

\section{Declaration}

1. I hereby state that the paper being submitted has not been published, simultaneously submitted, or already accepted for publication elsewhere.

2. This manuscript has been read and approved by all the authors, the requirements for authorship have been met, and each author believes that the manuscript represents honest work.

3. To the best of my knowledge, this work does not infringe upon any copyright or property right of any third party.

4. We have no financial interest/ conflict of interest.

\section{References}

1. Chakravarti T, George S (2013) An Overlap Syndrome of Pigment Dispersion and Pigmentary Glaucoma accompanied by Marfan Syndrome: Case Report with Literature Review. J Current Glau Prac 7(2): 91-95.

2. Farrar SM, Shields MB (1993) Current concepts in pigmentary glaucoma. Surv Ophthalmol 37(4): 233-252.

3. Andersen IS, Pralea AM, DelBono EA, Haines JL, Gorin MB, et al. (1997) A gene responsible for the pigment dispersion syndrome maps to chromosome 7q35- q36. Arch Ophthalmol 115(3): 384-388.

4. Scheie HS, Cameron JD (1981) Pigment dispersion syndrome: a clinical study. Br J Ophthalmol 65(4): 264-269. 
(c) (i) Creative Commons Attribution 4.0 International License

For possible submissions Click Here

MSOR

Medical \&

Ophthalmolog
Research

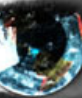

$\$ 2$

1.

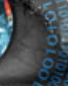

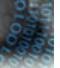

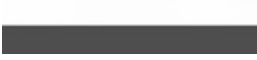

\section{Medical \& Surgical Ophthalmology Research}

\section{Benefits of Publishing with us}

- High-level peer review and editorial services

- Freely accessible online immediately upon publication

- Authors retain the copyright to their work

- Licensing it under a Creative Commons license

- Visibility through different online platforms 